Einen solchen Namen besitzen wir in dew n. pr. $\square \square$ 迎 ${ }^{1)}$ P-e dje-Pp, welchen ich nur in der Dyn. XXVI nachweisen kann. ${ }^{2}$ ) Die Bildung des Namens ${ }^{3}$ ) lässt keinen Zweifel daran, dass $\square$ Pp ein Göttername sein muss. Einen Gott $\mathrm{Pp}$ kennt das aegyptische Pantheon nicht, wohl aber ist dieser Name der eines bekannten Herrschers der Dyn. VI, den die Griechen als $D_{\iota} \omega \psi$ wiedergegeben haben. Also mit dem Namen des göttlish verehrten Phiops ist unser n. pr. in der Dyn. XXVI neu gebildet worden, und damit erhalten wir den sicheren Beweis, dass der Kultus dieses Herrschers in der Saitenzeit ein volkstümlicher war. Wer einmal die theophoren aegyptischen Eigennamen auf die beregte Frage hin durchforscht, wird zweifellos noch andere volkstümliche GötterKönige so ermitteln können. Dass auch die herodoteischen Erzählungen über die alten Könige berücksichtigt werden müssen, bedarf kaum der Erwähnung. Wenn Cheops, Chephren und Mykerinos zu Herodots Zeit volkstümliche Gestalten waren, so wird doch wohl auch ihr Kultus volkstümlich gewesen sein. Wenigstens liegt dieser Schluss sehr nahe.

Strassburg i. E. 25. 6. 01.

\section{Iteration im Elamischen IV ${ }^{4}$ ).}

Von G. Hü sing.

Die Veröffentlichung der neuen Funde aus Susa, von denen soeben Tome III (Scheil: Textes Elamites-Anzanites, Iière série) erschienen ist, legt es nahe, meine Studien über Iteration und Reduplikation im Elamischen wieder aufzunehmen und nachzuprüfen.

Der neu erschienene Band bringt auch die Mal-Amirtexte in wesentlich verbesserter Anflage. Der Name Tahhihi kann nun als gesichert gelten, da nach Scheils Angabe ein unveröffentlicher "texte juridique " die Form Tahhuhu bietet. "Stellen wir dazu die Namen Štruru und Sumumu, so liefert Malamir allein schon 3 Namen dieser Art, wenn nicht etwa MA II 21 amma Kuku zu lesen ist, denn das vermeintliche raš von Malamir wird wohl eher ein $k u$ sein. Auch sonst

1) S. die varr. bei Bergmann in Recueil III/149 A.2. ) Rec. 3/149 Devéria: Catal. 102 Serapeum Stele 72 (ed. Chassinat).

) S. Spiegelberg: Demot. Studien I. S. 30.

4) vgl. 1899 Sp. 111 n. 1900 Sp. 82. könnte noch (z. B. ebenda 15) ein entsprechender Name in den Texten gefunden werden.

Die Auffassung dieser Namen als Kur\%formen stimmt auch völlig zu Wincklers Verbesserung mấr ahat ša Šilhaha (OLZ $1901 \mathrm{Sp}$. 448): ein Ninšilhaha wäre auffallend.

Im übrigen enthalten die Texte Götternamen wie Belala (vgl. Bilala, Bulala, Alala, Tome III S. 29) LU-LU, MA-MA, EN$G A L-D U-D U$, die, oder deren verdoppelte Silbenzeichen, wohl auch phonetisch zu lesen sein mögen. Dazu gehört wohl auch $\check{C}_{a-m a} a(l)-m a(l)$, das an das íana von Malamir erinnert.

Die letzteren Namen entstammen bereits dem Obeliske des Maništusu, von dessen Personennamen eine Probe folge:

Abubu, Adada, Ahuhu, Allala, Allulu, Ištutu, Edada, Ekıtku, Dambaba, Čllala, Ćallulu, UR-UR, ZU-ZU, ZI-ZI, Dada, Kamama, MIEŠIS̆LS̆LS̆, Ibbubu u. s. w.

Bezeichnend ist der gleiche Vokal der verdoppelten Silbe. Unter diesen Namen kommt auch ein $K u-k u$ vor, allerdings als Mannesname, aber bisher fehlt ja jede Spur einer Unterscheidung männlicher und weiblicher Namen.

Aber die neuen Texte weisen auch neue Verbalformen auf.

So ein tahha-h (XXX 4), wie es freilich vorauszusetzen, aber eben noch nicht belegt war. Das Wort ist natürlich auf der ersten Silbe betont, es ist also einleuchtend genug, dass die Form sich zu (tahh), tah verkürzte, wie es z. B. LXIIII und XXIII 4 vorkommt. So auch S. 86 Z. 4, wo es Z. $4 / 5$ durch ta-at-tah variiert wird, das in dem neuelamischen Texte Sutur-Nahhuntes des Dritten als $d a-a d-d a-h$ geschrieben vorliegt. Dieser Text endigt auf $d a-a k-n i \quad(=t a-k-n e)$, in Malamir tak-ni geschrieben (in I) neben $d a-a k-n i$ (in II). Ausserdem in Malamir I 10 tah-ha-ak-ni. Die $\sqrt{t a h}$ ist also za ta vor den Endungen verkürzt, wenn der nthematische ${ }^{*}$ Vokal fehlt; man denke sich nur ein $t a h-h, t a h-h, t a h-s$ schnell ausgesprochen!

Die Revision der Malamirtexte bestätigt die Lesung da-ha-tah-a-iš-ni (taha-tah-a(i)-sne) in MA II 28. Sollte nicht zu beachten sein, dass auch hier wie im obigen tattah die iterierte Form nach der einfachen (Z. 24, $25,26)$ gebraucht wird?

So ist also tattah assimiliert aus tah-tah- $h$, wie die neue Form kikkiteh (XX 8) wohl aus kit-kite-h entstanden zu erklären ist. Auch 
die Malamirformen kite-kkite-k und kite-kte$m$-pe werden bestätigt, wie auch die einfache Form kite-m-pe.

Ferner findet sich ein kuktunra (XXV 2), das ja offenbar vom neuelamischen louktanta nicht zu trennen ist. Das Objekt zu kukktanra ist nun sijan, das hier (als Örtlichkeit) ebenso einen wagerechten Keil vor sich hat, wie z. B. kilam in Sutruk-Nahhunte C. 11. Das Ganze scheint eine Art Apposition zu Untaš-Riša zu sein, und diesen, da kukti= "schützen", als den Beschützer des Tempels zu bezeichnen. Nun kommt aber sehr oft an entsprechender Stelle ein kukra oder kukpa vor (je nachdem ein oder mehrere Götternamen davor genannt sind), das Scheil mit ${ }_{n}$ maître(s) du temple" zu übersetzen versucht. Dieses sijan kukra scheint weiter auch an Stelle des oft belegten sijan $k u k$ vorzulommen (vgl. XIV 4 und Variante), und $k u k$ kehrt wieder in den Namen $K u k$ Kirpiaš und $K_{u} \iota k$-Našutaš. In diesen Namen könnte es gar wohl "Schützling" bedeuten, als "passives" Particip, zu dem kukra das "nomen agentis" sein kann, wie ja auch ein kukme ${ }^{1}$ ) belegt scheint (XIX 4). In den Textes Élamites-Sémitiques S. 74 erinnert Scheil auch an die (kassische) Glosse kukla $=a r d u$, was wohl zutreffend sein kann, ohne dass ardu eine wörtliche Übersetzung sein brauchte.

Bei obiger Annahme wäre freilich ein sijan kuk nicht zu erklären; da aber an allen Stellen ein $\mathbf{z w e i t e s}$ sijan folgt, so könnte kuk zu diesem gehören. Die betreffenden Stellen wären dann abzuteilen (vgl. XIII u. XV):

sijan, kuk sijan-i-me . . . kušǐ.

sijan, kuk sijan-ra ir-mur-tah.

sijan, kuk ulluruk, sijan kukra (humah?)

(Die Variante zu XIV ist verschrieben: hinter kulk fehlt sijan, dafür ist murtah doppelt geschrieben.)

kuck kann aus"leutk entstanden sein, so wäre der Zusammenhang zwischen kuk-ra und kuktan-ra herzustellen.

Dass $\sqrt{k u t}$ nicht bloss „bringen", sondern etwa in seine Obhut nehmen" bedeutet, zeigt der Text auf der Naram-Sin-Stele (SutrukNahbunte $D=$ XXIV bei Scheil). Hier lautet die Verbalform kuthi, in Sutruk-Nahhunte C kutha, in Malamir I 11 kutuh ${ }^{2}$ ) (ev. kutoh), ebenso nach Scheil in II 2, womit dann wohl auch kutor zusammenhängen wird.

1) Dahinter ku-si-h! Scheil hat offenbar seinen Augen nicht getraut!

i) Vgl. ku-toó-ta (II 34), Konjektur Borke, jetzt durch soheil bestrtigt.
Jedenfalls wird kukti als Iteration von kuti durch die neuen Funde eher bestätigt, als angefochten.

Auch weitere Formen mit pepši finden sich; in Malamir I 15 aber steht pipsin (nicht piših!)

Dagegen steht ebenda II 32 pi-ip-tu(m) šahiš und bald darauf pitte-ma-n-ra. In I 11 dürfte auch pe-pte-na als erste Person zu fassen sein, wie das folgende $u$ (!) kine$n a$, in I 23 aber huma-k nete-na, wie auch II 31 Nahhanti nete-na abzuteilen scheint ${ }^{1)}$. Als Bedeutung für peptena dürfte "schmücken" (vgl. pitte, OLZ. $1901 \mathrm{Sp} .446 / 7$ - II 22 steht nicht pittinra!) sehr wohl passen. Scheil LVIII 15 steht $u$ pi-tah-ha, worauf pittes folgt.

Eine der wunderlichsten neuen Formen ist die Variante hu-ut-šu-tak für huttak. Stünde hier statt $\grave{s} u$ (kat) eine Lücke, so würde ich das fehlende Zeichen unwillkürlich hut lesen. Leider ist mir zur Zeit Brünnows Zeichenliste nicht zugänglich, doch hat kap auch den Wert hup; könnte kat den Wert hut haben?2) Dann könnte in $K u k$-(nap)- $\mathrm{Na}$ $\check{s ̌ u t a s ̌ ~ e i n ~ K u k a n n a-h u t t a s ̌ ~(=~}{ }_{\text {Er }}$ hat das kukunnum gemacht") und in $K u k$ (nap) Kirpiaš ein Kukan-kirpi-iaš stecken. Ein sumerischer Text schreibt freilich $K u-u k$ kir-pi-aš, allein solch kunstvolle Texte mahnen zur Vorsicht, und man denke an den Gott Duniaș!

$\mathrm{Ob}$ sich noch weitere für unsere Frage bemerkenswerte Formen finden lassen, das kann erst die allmähliche Verarbeitung des neuen Stoffes zeigen; Scheils Vocabulaire ist wegen der oft so schwierigen Worttrennung dazu nicht ausreichend. Immerhin sind schon jetzt die Formen su-us-qa-tah und $z u-u q-q a-t a h$ beachtenswert, wie sie auch zu erklären sein mögen. Sie zeigen doch wohl, dags ein $z$ vor $k$ im Sandhi verschwinden konnte.

Vielleicht ist es angebracht, hier auch darauf hinzuweisen, dass nach Weissbachs Besserung seines $t u$ in $l a$ nun auch kutkalarraki u. s. w. zu lesen wären, was Foy nicht minder übersehen hat (WZKM 14 S. 285) als ich. (Ebenso appanlakkime, das dann natïrlich nichts mit huh-pe-n-tukkime zu thun hat). Indessen bliebe wohl immer noch abzuwarten, ob eine genaue Revision der Bagistaninschrift nicht gar noch die drei

1) Doch scheint ein Weib Ammatena (oder amma Tena?) zu heissen (Bcheil S. 112), wenn nicht etwa tena possessiv ist: ten keh "ich nahm von ihm (von dort) weg?"

3) (Bei Brünnow nioht verzeichnet. D. R.) 
fehlenden Keile (Zeichen to $=12$ bei Weissbach) auffindet. Was Zeile 45 verlesen war, konnte leicht auch Zeile 47 verlesen werden. Man vergleiche nur den verbesserten Text der Malamirinschriften mit dem bisherigen!

Eine umfassende Besprechnng der neuen Gabe Scheils wird folgen; die Vorwegnahme des Obigen mag dazn beitragen, die Besprechung zu entlasteu.

\section{Bespreehungen.}

Eduard Sievers, Metrische Studien I: Studien zur hebräischen Metrik; 1. Bd. Untersuchgg. 2. Bd. Textproben. Leipz. 1901. Y.. $12+6$ Mark. Besprochen von Hubert Grimme.

E. Sievers' Metrische Studien bedeuten ein Ereignis für die hebräische Wissenschaft. Vielleicht wird man einmal von ihrem Erscheinen die Periode datieren lassen, da der Gedanke einer hebräischen Metrik endgiltig zum Gemeingute der Bibelgelehrten wurde; sicher aber werden viele der darin aufgeworfenen Fragen nicht ungehört und unbeantwortet verhallen dürfen. In einer Zeit erschienen, da die bebräische Sprach. wissenschaft in grosser Selbstzufriedenheit und Rube scheinbar fertig und grundtief sich darstellt, wirkt das Buch wie ein Steinwurf in stilles Wasser: die glatte Oberfläche ist zerstört und der $\mathrm{Z}_{\text {weifel zieht seine weiten }}$ Kreise über der aufgeregten Tiefe. Dass eg so gekommen ist, erfüllt mich mit Befriedigung, die dadurch verdoppelt wird, weil der Anstoss von indogermanischer Seite erfolgt, und so jetzt endlich zwischen den beiden so lange getreunt gewesenen Richtungen der Sprachwissenschaft, der indogermanischen und semitischen, eine Annäherung sich vollzieht, die allerdings vor der Hand nicht sehr brüderlich aussieht. Ich hoffe, dass die Semitistik Veranlassung nimmt, kräftig auf den Stoss, der gcgen sie geführt ist, zu reagieren, andrerseits aber von dem Wagemute des Angreifers sich soviel absieht, dass sie im eigenen Hause vor grossen Umgestaltungen fürderhin nicht mehr zurückschreckt.

Dass die Metrischen Studien ein Wagnis darstellen, dessen ist sich $\mathrm{E}$. Sievers selbst wohl bewusst. Seine ganze Vergangenheit gehört der Beschäftigung mit indogermanischer Grammatik und Metrik, wenn wir davon absehen, dass er einer der Begründer der exakten Phonetik ist. Zum Hebraisten hat ihn eigentlich erst der Augenblick gemacht, da er - es war im Anfange des Jahres 1898 - auf eine Anregung von
Fr. Buhl hin einige anscheinend regelmässig gebauten Stücke hebräischer Poesie durchskandierte und in ihnen anapästischen Rhythmus $z u$ entdecken glaubte. Von diesen Augenblicke an hat ihn die hebräische Metrik mit ihren hunderterlei Konsequenzen wie in einem Zauberbann gehalten, aus dem er sich er.st durch das Erscheinen der beiden stattlichen Bände lösen konnte, deren erster die metrischen und grammatischen Untersuchungen enthält, während der zweite auf mehr als 150 Seiten rhythmisierte Textproben mit Transskription bietet.

Es ist die grösste Arbeit, die bisher über hebr. Metrik erschienen ist, ganz aus dem Vollen gearbeitet, dazu voll Begeisterung und Frische. Verfasser erweist sich als grimmigen Gegner grauer metrischer Theorien, die sich vielleicht auf dem Papiere gut uusnehmen, aber nicht an das $\mathrm{Ohr}$ appellieren. Seine höchste Instanz ist das Gehör: 'Dle korrelkt durchgeführte Leseprobe entscheidet' sagt er, und was am besten klingt, dem bietet er den Preis der Richtigkeit. Wie er selbst, so soll auch jeder Leser die Probe durchs Gehör machen und, um sie ihm zu erleichtern, transskribiert er alle seine Beispiele. Sein Vorgehen darin ist sehr bemerkenswert. Die Konsonanten boten in den 6 teils mit, teils ohne Dageš lene geschriebenen Buchstaben eine ernsthafte Schwierigkeit: soll man ihre unpunktierte Form durch Aspiraten oder Spiranten wiedergeben? S. entscheidet sich kurzerhand für die Spiranten, die er dann mit den Zeichen der gleichen altgermanische Laute umschreibt. Dadurch scheint mir aber die Frage noch nicht gelöst zu sein. Den alexandrinischen Uebersetzern der Bibel war sicher $\mathrm{J}=\mathrm{kh}$, da sie die in Aegypten nur kh und ph gesprochenen $x$ und $\varphi$ (vgl. Indogerm. Forschgg., VI, 123 ff.: J. J. Hess, Zur Aussprache des Griechischen) zur Transskription von I und 9 benutzten. Liegt ferner wicht eine grosse Unwahrscheinlichkeit darin, dass die hebr. Spiranten bei der Gemination zu Explosiven werden müssen, also såfar im $\mathrm{Pi}^{\dagger}$ el zu sippar, während doch z. B. das Arabische geminierte Spiranten vollauf verwendet? Endlich welch seltsamer Lautwechsel, wenn z. B. zächär nach Anhängung von -on zunächst zikkårōn wird, von dieser Form aber der Stat. constr. sichrōn zur Spirans zurückkehrt! Alle Schwierigkeiten werden gehoben bei der Annahme, dass die unpunktierten Buchstabenformen wirkliche Aspiraten darstellen, also z. B. $\Xi=\mathrm{kh}$ ist, die Verdopplung aber aus ihm kkh macht ( $\left.\vee g l .9 y=A x \chi^{\omega}\right)$, somit 'åkhal'ukkhal, zäkhär' 